\title{
Análisis crítico de la fiscalidad de las cooperativas de segundo grado como fórmula de integración ${ }^{1}$
}

\author{
Marina Aguilar Rubio²
}

Recibido: 23 de agosto de 2017 / Aceptado: 8 de noviembre de 2017

Resumen. Las sociedades cooperativas de segundo grado constituyen uno de los instrumentos básicos de concentración empresarial previstos en la legislación cooperativa y ha sido la fórmula tradicionalmente utilizada por las cooperativas a la hora de acometer un proyecto de integración.

El trabajo que se presenta trata de mostrar la especial tributación de las sociedades cooperativas de segundo grado, que nos interesan especialmente por ser el instrumento de integración más utilizado en España por las sociedades cooperativas para emprender procesos de concentración en el ámbito agrario, sector clave en nuestro entorno geográfico.

Centraremos el estudio de su fiscalidad en el Impuesto sobre Sociedades por las particularidades tributarias que presenta el mismo en este tipo societario. Las sociedades cooperativas de segundo grado pueden optar por tributar como simples sociedades cooperativas, según lo regulado en la Ley 20/1990, de 19 de diciembre, sobre Régimen Fiscal de Cooperativas, o hacerlo como grupo cooperativo, acogiéndose en el régimen especial de tributación consolidada establecido para este tipo social.

Al igual que las sociedades de primer grado éstas disfrutan de una serie de beneficios fiscales tanto en la imposición directa como en la indirecta, como consecuencia de la naturaleza social de las mismas.

Palabras clave: Tributación; Intercooperación; Integración; Dimensión; Cooperativas de segundo grado; Grupos cooperativos.

Claves Econlit: F36; H29.

\section{[en] Critical analysis of the taxation of second-tier cooperatives as integration formula}

\begin{abstract}
Second-tier cooperatives are one of the basic instruments for business concentration in the cooperative law and the formula traditionally used by cooperatives when it comes to building an integration project.

The work tries to show the special taxation of the second-tier cooperatives, that we are interested especially because it is the instrument of integration most used in Spain by cooperatives to undertake concentration processes in the agri-food field, key sector in our geographical environment.

We will focus the study of its taxation in the Corporate Tax due to the tax peculiarities that it presents in this type of company. Second-tier cooperative societies may choose to tax as simple cooperative societies, as regulated in Law 20/1990, of December 19, on Cooperative Tax Regime, or to make as a

1 Este trabajo se ha realizado en el marco del Proyecto de Investigación de Excelencia de la Junta de Andalucía "Retos y oportunidades en la concentración e integración de empresas agroalimentarias (P12-SEJ- 2555)" del que la autora es miembro investigador

2 Universidad de Almería, España
\end{abstract}

Dirección de correo electrónico: magui@ual.es 
cooperative group, accepted in the special regime of consolidated taxation system established for this social type.

Keywords: Taxation; Intercooperation; Integration; Dimension; Second-tier cooperatives; Cooperative groups.

Sumario. 1. Introducción: La integración de las cooperativas a través de cooperativas de segundo grado y de los grupos cooperativos. 2. Régimen fiscal de las sociedades cooperativas en España. 3. Las cooperativas de segundo grado en el impuesto sobre sociedades. 4. Los grupos cooperativos en el impuesto sobre sociedades. 5. Conclusiones. 6. Referencias bibliográficas.

Cómo citar: Aguilar Rubio, M. (2017) Análisis crítico de la fiscalidad de las cooperativas de segundo grado como fórmula de integración. REVESCO. Revista de Estudios Cooperativos, Monográfico, $\mathrm{N}^{\circ}$ 126, pp. 118-132. DOI: 10.5209/REVE.58393.

\section{Introducción: La integración de las cooperativas a través de cooperativas de segundo grado y de los grupos cooperativos}

Las técnicas de concentración empresarial son una herramienta importantísima para las empresas en el contexto actual de mercados globalizados. Las sociedades cooperativas no son ajenas a esta tendencia a la globalización y a los retos de los mercados cada vez más competitivos, por lo que se hacen necesarias formas empresariales de mayor dimensión, más eficientes y dinámicas, que permitan mejorar la competitividad y asegurar la supervivencia.

El término integración tiene en el cooperativismo entidad propia frente al de concentración empresarial con el que se denominan las parecidas técnicas que utilizan las sociedades de capital para crear empresas de gran tamaño. Se quiere así subrayar, de un lado, que las cooperativas se integran pero conservan su individualidad $\mathrm{y}$, de otro, que estas realidades grupales tienen en Derecho cooperativo particularidades jurídicas y económicas propias (Embid Irujo, 1998:8). Si la fusión supone una total integración, económica y jurídica de las entidades que participan en ella —concentración en la unidad-, la integración cooperativa mantiene las unidades que se agrupan — concentración en la pluralidad- (Alfonso Sánchez, 2000: 25). Evidentemente, las opciones de integración empresarial no están exentas de riesgos. En cuanto afectan a las cooperativas, se han señalado, como más destacados, el distanciamiento de la base social y la burocratización y obsolescencia del proyecto (Rosembuj, 1988: 169). Ello, no obstante, parecen más convenientes que problemáticas y, en algunos sectores, como el agroalimentario, resultan imprescindibles no solo para el progreso sino, incluso, para la propia supervivencia en los nuevos mercados.

Las cooperativas de segundo grado (CSG) constituyen el mecanismo por excelencia de integración cooperativa, con una individualidad propia, regulación peculiar en las leyes cooperativas y con una larga tradición en determinados sectores del cooperativismo. Éstas son sociedades cooperativas compuestas por dos o más cooperativas (aunque se admite, por lo general, la participación como socios de otro tipo de entidades) que se unen con objeto de promover, coordinar y desarrollar fines económicos comunes de las entidades socias e integrar la actividad económica de las mismas. La flexibilidad que ofrece este peculiar subtipo social cooperativo permite alcanzar mayores o menores cuotas de integración 
dependiendo de la voluntad de sus miembros, que van desde facilitar meras colaboraciones empresariales hasta servir de marco organizativo a verdaderos fenómenos grupales a los que el legislador le da una específica vestidura jurídica (Embid Irujo, 1998: 167).

En el art. 77.1 de la Ley 27/1999, de 16 de julio, de Cooperativas (en adelante LCOOP), se definen como cooperativas que se constituyen por, al menos, dos cooperativas y aunque pueden integrarse en calidad de socios otras personas jurídicas y empresarios individuales el conjunto de éstos sólo puede llegar hasta un máximo del cuarenta y cinco por ciento del total de los socios.

Aunque tienen por objeto promover, coordinar y desarrollar fines económicos comunes de sus socios, y reforzar e integrar la actividad económica de los mismo, la regulación actual de las CSG en las leyes cooperativas se caracteriza por la amplitud funcional, por lo que la colaboración empresarial podrá ser tan intensa como admitan las entidades que las integran, aunque sin llegar a anular o prescindir de la fisonomía de cada miembro agrupado. En cada caso, serán los estatutos los que establezcan el grado de vinculación entre las entidades participantes, pudiendo ésta limitarse a una mera colaboración o a alcanzar una concentración empresarial en sentido propio. Como se ha señalado, queda reservada a la autonomía de la voluntad, consignada en los estatutos, la determinación concreta del modo de vinculación querida, así como su grado de intensidad (Vargas Vasserot, Gadea Soler y Sacristán Bergia, 2017: 229).

La creación de cooperativas de segundo o ulterior grado ha sido la fórmula de cooperación más utilizada por las cooperativas, en especial en el ámbito agrario: en primer término, por la tradición normativa y doctrinal (todas las legislaciones que inciden en la materia cooperativa regulan esta fórmula como un modelo propio de organización empresarial cooperativa); $\mathrm{y}$, en segundo término, por una denominada interpretación "purista" de algunos principios cooperativos, como el segundo, de control democrático, que no parecía casar con otras fórmulas de integración cooperativa más profundas como las fusiones (Peris García, 2002: 71).

Esta visión ha sido ampliamente superada y no podemos desconocer que el sexto principio cooperativo viene a establecer que estas sociedades sirven mejor a sus socios y al propio movimiento cooperativo trabajando conjuntamente mediante estructuras locales, regionales, nacionales e internacionales. Este principio de cooperación entre cooperativas ha sido recogido de manera implícita o explícita en todas las leyes sustantivas que regulan las sociedades cooperativas en España.

La realidad de las CSG se ve afectada, como no, por el panorama legislativo español en materia de cooperativas, donde coexisten dieciséis leyes autonómicas aplicables exclusivamente en su territorio, la ley de sociedades cooperativas estatal, de carácter supletorio, además de algunas leyes consideradas sectoriales. En la medida en que no es posible un análisis pormenorizado de cada una de las normas que inciden en la cuestión, respecto de los aspectos sustantivos, hemos optado por centrar el análisis en la citada LCOOP.

La regulación actual de las CSG en nuestras Leyes más modernas se caracteriza por la amplitud funcional, por lo que la colaboración empresarial podrá ser tan intensa como admitan las entidades que las integran, aunque sin llegar a anular o prescindir de la fisonomía de cada miembro agrupado (Gadea Soler, 2010: 252). En cada caso, la determinación concreta del modo de vinculación querida, así como su 
grado de intensidad, queda reservada a la autonomía de la voluntad en el marco de la amplia configuración del fin social establecido por el legislador (Embid Irujo, 1998: 227), por lo que serán los estatutos los que establezcan el grado de vinculación entre las entidades participantes, pudiendo ésta limitarse a una mera colaboración o a alcanzar una concentración empresarial en sentido propio (Alfonso Sánchez, 2000: 269, Gadea Soler, 2010: 252).

Este trabajo tiene como objeto analizar la fiscalidad de las CSG, centrándonos en el Impuesto sobre Sociedades (en lo sucesivo IS). Ello exige estudiar la situación de que actúe como CSG propiamente dicha y también el supuesto de que desarrolle su actividad como cabeza de un grupo cooperativo. En cualquier caso, esto supone acercarnos a dos realidades diferentes:

Por una parte, analizaremos las CSG como fórmula de cooperación, entendiendo por tal toda actuación tendente a la colaboración entre empresas pero salvaguardando la independencia de las mismas. Ello sin olvidar que, como ya hemos adelantado, se podría por esta vía alcanzar una auténtica concentración empresarial.

Por otra parte, nos vamos a centrar en los grupos de sociedades, en este caso cooperativas, como uno de los exponentes de la concentración empresarial, aunque no suponen la desaparición de la personalidad jurídica de las sociedades agrupadas - como sí sucede en los procesos de fusión, absorción o escisión-, pero sí una unidad de decisión, que puede ser más o menos centralizada por la entidad dominante.

\section{Régimen fiscal de las sociedades cooperativas en España}

El régimen fiscal aplicable a las cooperativas se recoge en la Ley 20/1990, de 19 de diciembre, sobre Régimen Fiscal de Cooperativas (en adelante LRFC). En lo no previsto en la misma, les resulta de aplicación la Ley 27/2014, de 27 de noviembre, del Impuesto sobre Sociedades (en lo sucesivo LIS).

La LRFC confiere a las cooperativas un tratamiento fiscal diferenciado en la medida que establece una forma de tributación que regula:

- por una parte, medidas de ajuste técnico que adaptan los preceptos tributarios a las especiales características de las cooperativas (aportaciones de los cooperativistas, rendimientos de los cooperativistas, retornos, etc.) y

- $\quad$ por otra parte, determinados beneficios fiscales, tanto en la imposición directa como en la indirecta.

Las primeras en modo alguno pueden ser consideradas beneficios, ya que, si bien pueden suponer una ventaja en relación con las que integran el régimen general, no tienen una finalidad incentivadora, sino una necesidad técnica aplicativa del tributo. Por ello resultan de aplicación incluso a las cooperativas que no gozan de protección fiscal a los efectos de la LRFC, esto es, aquéllas que no se constituyan con arreglo a los principios y disposiciones de la Ley sustantiva que las rija o que incurran en alguna de las causas de pérdida de la condición de cooperativa fiscalmente protegida reguladas en el art. 13 de la citada norma. 
Según el art. 6 LRFC, serán consideradas como cooperativas protegidas «aquellas entidades que se ajusten a los principios y disposiciones de la LCOOP o de las leyes de cooperativas de las Comunidades Autónomas que tengan competencia en esta materia y no incurran en ninguna de las causas previstas en el artículo 13». Este precepto será objeto de análisis en sede del apartado dedicado al estudio del régimen fiscal de las CSG.

Por su parte, se incluyen en el grupo de las especialmente protegidas las cooperativas de primer grado que, con ciertas condiciones y requisitos, pertenezcan a alguna de las siguientes categorías: de trabajo asociado; agrarias, de explotación comunitaria de la tierra; del mar; o de consumidores y usuarios (art. 7 LRFC).

A continuación, vamos a recoger los beneficios fiscales establecidos en la LRFC para el Impuesto sobre Sociedades en las cooperativas protegidas.

- $\quad$ Tipo de gravamen (art. 33.2 LRFC):

a. Para los resultados cooperativos, el tipo aplicable es del $20 \%$.

b. Para los resultados cooperativos de las cooperativas de crédito, el tipo es del $25 \%$.

c. Los resultados extracooperativos se gravan al tipo general del Impuesto, que actualmente es el $25 \%$.

- Libertad de amortización: según el art. 33.3 LRFC, gozarán de libertad de amortización de los elementos de activo fijo nuevo amortizable, adquiridos en el plazo de tres años a partir de la fecha de su inscripción en el Registro correspondiente.

Compensación de pérdidas: Establece art. 23 LRFC que la suma algebraica de las cantidades resultantes de aplicar a las bases imponibles los tipos de gravamen constituye la cuota íntegra, siempre que sea positiva. Esta última precisión resulta necesaria porque la compensación de pérdidas se realiza para las cooperativas en la cuota y no en la base imponible como sucede con carácter general en el Impuesto sobre Sociedades. Así, si la suma algebraica de las cuotas resultase negativa, su importe podrá compensarse por las cooperativas con las cuotas íntegras positivas con el límite del 70\% de la cuota íntegra previa a su compensación. En todo caso, serán compensables en el período impositivo cuotas íntegras por el importe que resulte de multiplicar un millón de euros al tipo medio de gravamen de la entidad.

Cuota tributaria: En general, hay que entender que resultan aplicables a las cooperativas las deducciones de la cuota establecidas con carácter general en la LIS. Ahora bien, existen ciertas especialidades: por ejemplo, la cuantía de la deducción para evitar la doble imposición de dividendos e internacional se obtendrá aplicando el tipo de gravamen que corresponda en función del carácter cooperativo o extracooperativo de los rendimientos que originan dicha deducción.

A las sociedades cooperativas calificadas como especialmente protegidas, además, de los beneficios fiscales anteriormente enumerados, se les aplica una bonificación 50\% de la cuota íntegra del impuesto (art. 34.2 LRFC).

Los beneficios que afectan a otros impuestos se sintetizan en la tabla: 
Tabla. 1.

\begin{tabular}{|c|c|c|}
\hline & Protegidas & $\begin{array}{l}\text { Especialmente protegidas } \\
\text { Todas las anteriores y: }\end{array}$ \\
\hline \multicolumn{3}{|c|}{ IMPUESTOS DIRECTOS (locales): } \\
\hline $\begin{array}{l}\text { Impuesto sobre } \\
\text { Actividades } \\
\text { Económicas }\end{array}$ & $\begin{array}{l}\text { Bonificación del 95\% sobre cuota } \\
\text { y recargos }\end{array}$ & \\
\hline $\begin{array}{l}\text { Impuesto sobre } \\
\text { Bienes } \\
\text { Inmuebles }\end{array}$ & $\begin{array}{l}\text { Bonificación del 95\% sobre cuota } \\
\text { y recargos, para sociedades } \\
\text { cooperativas agrarias y de } \\
\text { explotación comunitaria }\end{array}$ & \\
\hline \multicolumn{3}{|c|}{ IMPUESTOS INDIRECTOS: } \\
\hline $\begin{array}{l}\text { Impuesto sobre } \\
\text { Transmisiones } \\
\text { Patrimoniales y } \\
\text { Actos Jurídicos } \\
\text { Documentados } \\
\text { (estatal cedido a } \\
\text { las Comunidades } \\
\text { Autónomas) }\end{array}$ & $\begin{array}{l}\text { Exenciones: } \\
\text { Transmisiones Patrimoniales } \\
\text { Onerosas: } \\
\text { Adquisiciones de bienes y } \\
\text { derechos que se integran el Fondo } \\
\text { de educación y promoción } \\
\text { Operaciones Societarias: } \\
\text { Actos de constitución, ampliación } \\
\text { de capital fusión y escisión } \\
\text { Constitución y cancelación de } \\
\text { préstamos } \\
\text { Actos Jurídicos Documentados: } \\
\text { Adquisiciones de bienes y } \\
\text { derechos cuando, por tratarse de } \\
\text { operaciones sujetas a IVA, se } \\
\text { someterían a gravamen por este } \\
\text { tributo de no mediar tal exención }\end{array}$ & $\begin{array}{l}\text { Adquisiciones de bienes y } \\
\text { derechos destinados } \\
\text { directamente a cumplir sus } \\
\text { fines sociales y estatutarios }\end{array}$ \\
\hline
\end{tabular}

Fuente: Elaboración propia

Las incesantes reformas llevadas a cabo en las leyes españolas de cooperativas no se han visto acompañadas por una reforma en el régimen fiscal en el mismo sentido. A título de ejemplo, la LCOOP se aprobó nueve años después de la LRFC, pero es que la tercera generación de leyes autonómicas pertenece a la década actual. Las consecuencias de este «divorcio» se resumen en que, por un lado, las cooperativas que cumplen los requisitos para ser fiscalmente protegidas se encuentran en peor situación en términos de mercado; y por otro, las cooperativas que intentan aprovechar las mejoras de su régimen sustantivo que les ofrecen sus legislaciones autonómicas corren el riesgo de perder los beneficios fiscales previstos en la LRFC (Rodrigo Ruiz, 2010: 9-25). Ello unido a la atenuación de la tributación de las sociedades de capital en España da como resultado que pueda resultar más beneficioso para ellas aplicar el régimen general (Tejerizo López, 2010: 75). 


\section{Las cooperativas de segundo grado en el impuesto sobre sociedades}

Todo el régimen fiscal de las CSG se regula en un solo artículo de la LRFC: el art. 35. Este artículo distingue los diferentes niveles de tratamiento fiscal que pueden tener las CSG y que reproducen el esquema de las de primer grado. Veámoslo:

\subsection{Cooperativas de segundo grado protegidas}

Las cooperativas de segundo y ulterior grado que no incurran en ninguna de las circunstancias señaladas en el art. 13 de esta Ley disfrutarán de los beneficios fiscales previstos en el art. 33.

Las causas de pérdida de protección fiscal se pueden sintetizar así:

Causas relativas a los fondos cooperativos: no hacer las dotaciones al Fondo de Reserva Obligatorio (FRO) y al Fondo de Educación y Promoción (FEP) en los supuestos, condiciones y cuantías previstas en las disposiciones cooperativas; repartir tanto el activo sobrante en el momento de la liquidación de la Cooperativa como los fondos que tengan carácter de irrepetibles entre los socios durante la vida de la sociedad; y aplicar a finalidades distintas de las previstas por la Ley, cantidades del FEP.

- Causas relativas a los socios: acreditar a los socios en proporción distinta a las entregas, actividades o servicios realizados con la cooperativa o distribuirlos a terceros no socios; exceder de los límites legales autorizados las aportaciones al capital social de los socios; o tener un número de socios inferior al previsto en las normas legales, sin que se restablezca en un plazo de seis meses.

Causas producidas por incumplimiento de normas substantivas: incumplir la normativa reguladora del destino del resultado de la regularización del balance de la cooperativa y del resultado de la actualización de las aportaciones de los socios al capital social; retribuir las aportaciones de los socios o asociados al capital social con intereses superiores a los máximos autorizados o superar tales límites en el abono de intereses por demora en el supuesto de reembolso de dichas aportaciones o por los retornos cooperativos devengados y no repartidos, por incorporarse a un Fondo Especial constituido por acuerdo de la Asamblea General; no imputar las pérdidas del ejercicio económico o imputarlas vulnerando las normas establecidas en la Ley, los Estatutos o los acuerdos de la Asamblea General; realizar más del $50 \%$ de las operaciones cooperativizadas de la cooperativa con terceros no socios, fuera de los casos establecidos en la Ley; contratar trabajadores asalariados en número superior al que autoriza la ley; y la falta de auditoría externa en los casos señalados en las normas legales.

Otras causas: participar en el capital social de entidades no cooperativas en cuantía superior al 10\% (y más del $50 \%$ de los recursos propios de la cooperativa). La cuantía podrá ascender al $40 \%$ si la participación se produce respecto de entidades que realicen actividades preparatorias, complementarias o subordinadas a las de la propia cooperativa. Además, la participación podrá ser superior si el Ministerio de Economía y Hacienda lo autoriza; incumplir las normas sobre contabilización separada de 
operaciones con terceros no socios: reducir el capital social a una cantidad inferior a la cifra mínima establecida estatutariamente, sin que se restablezca en un plazo de seis meses; paralizar la actividad cooperativizada la actividad los órganos sociales durante dos años, sin causa que lo justifique; y concluir la empresa que constituye su objeto o la imposibilidad manifiesta de desarrollar la actividad cooperativizada.

\subsection{Cooperativas de segundo grado especialmente protegidas}

Las cooperativas de segundo y ulterior grado que asocien, exclusivamente, a cooperativas especialmente protegidas disfrutarán de los beneficios fiscales previstos para éstas.

La LRFC establece con minuciosidad cuáles son los requisitos y características que deben reunir las cooperativas que, dentro de las categorías indicadas anteriormente, son merecedoras de un trato especial. El denominador común de las cooperativas de esta categoría parece ser que respetan los principios cooperativos de un modo más perfecto, bien atendiendo a sectores primarios de la actividad económica o cubriendo necesidades básicas de los ciudadanos.

Hemos elegido ejemplificar la situación de las cooperativas especialmente protegidas reproduciendo las condiciones de acceso al mismo para las cooperativas agroalimentarias, puesto que éstas son las CSG más comunes

Según el art. 9 LRFC, se considerarán especialmente protegidas las cooperativas agroalimentarias que cumplan básicamente los siguientes requisitos:

1. «Que asocien a titulares de explotaciones agrícolas, forestales, ganaderas o mixtas, situadas dentro del ámbito geográfico al que se extienda estatutariamente la actividad de la cooperativa». Para las CSG significa que tiene que estar conformada por cooperativas especialmente protegidas que sean titulares de explotaciones agroalimentarias. No cabe aquí que asocien a otro tipo cooperativas ni a otra clase de entidades como pueden ser las sociedades agrarias de transformación.

2. Que en la realización de sus actividades agrarias respeten los siguientes límites:

«a) Que las materias, productos o servicios adquiridos, arrendados, elaborados, producidos, realizados o fabricados por cualquier procedimiento por la cooperativa, sean destinados exclusivamente a sus propias instalaciones o a las explotaciones de sus socios». Sus socios, en este caso, serán las cooperativas agrupadas. Sin embargo, se permite ceder a terceros no socios siempre que su cuantía, durante cada ejercicio económico, no supere el $50 \%$ del total de las operaciones de venta realizadas por la CSG.

«b) Que no se conserven, tipifiquen, manipulen, transformen, transporten, distribuyan o comercialicen productos procedentes de otras explotaciones, similares a los de las explotaciones de la cooperativa o de sus socios, en cuantía superior, durante cada ejercicio económico, al 50\% del importe obtenido por los productos propios». Esto es, la actividad cooperativizada con terceros no socios (que no sean las propias cooperativas asociadas a la CSG) no pueden superar ese límite, que también opera en las operaciones 
con terceros que realice cada una de las cooperativas socias. Debemos tener en cuenta que, en el momento que una de las cooperativas socias, incumpla este $\mathrm{u}$ otros requisitos, pierde la calificación fiscal de especialmente protegida, lo que supondrá que la CSG también la pierda.

3. «[...]Las cooperativas dedicadas a la comercialización y transformación de productos ganaderos, en las que se integren socios titulares de explotaciones de ganadería independiente, que el volumen de las ventas o entregas realizadas en cada ejercicio económico, dentro o fuera de la cooperativa, por cada uno de estos socios, exceptuados los Entes públicos y las sociedades en cuyo capital social participen éstos mayoritariamente, no supere el límite cuantitativo establecido en el Impuesto sobre la Renta de las Personas Físicas para la aplicación del régimen de estimación objetiva singular». Aunque se admitirá que haya socios cuyas bases imponibles o volumen de ventas superen los indicados, siempre que no excedan en su conjunto del $30 \%$ de las que correspondan al resto de los socios.

Este apartado tercero, introducido por la Ley 13/2013, de 2 de agosto, de fomento de la integración de cooperativas y de otras entidades de carácter agroalimentario (en adelante, LFIC), está pensando en la posibilidad de integración de las cooperativas a fin de ganar dimensión, como demuestra el siguiente párrafo del mismo: «A efectos de la aplicación de estos límites, cuando figuren como socios otras cooperativas o sociedades o comunidades de bienes, las bases imponibles o el volumen de ventas de éstas se imputarán a cada uno de sus socios en la proporción que estatutariamente les corresponda».

La LFIC, como su nombre indica, tiene como objetivo fomentar los procesos de fusión e integración de cooperativas agrarias y de otras entidades asociativas, para favorecer, a través de la concentración de la oferta, el redimensionamiento de dichas entidades, el incremento de la competitividad internacional de las empresas agroalimentarias españolas y el necesario reequilibrio de la cadena alimentaria (Cano Ortega, 2015: 60-85). El vehículo diseñado para lograr estos ambiciosos objetivos es el reconocimiento a ciertas entidades asociativas agroalimentarias como Entidades Asociativas Prioritarias (EAP), a las que se les dispensa de un trato preferencial para la obtención de subvenciones y ayudas públicas. La entidad ha de tener la forma jurídica de sociedad cooperativa, cooperativa de segundo grado, grupo cooperativo, SAT, Organización de Productores reconocida o ser una entidad civil o mercantil cuando más del $50 \%$ del capital social de la misma pertenezca a alguna de las entidades antes citadas [art. $1.3 \mathrm{y}$ art. 3.1, letra a)].

Ahora bien, hay que tener en cuenta que, para aplicar el tratamiento del art. 9.3 a CSG, los socios no pueden ser distintos a cooperativas de primer grado especialmente protegidas. Debería haberse flexibilizado el régimen del art. 35 para las CSG en el sentido de permitir otro tipo de socios como, por otra parte, también hacen las leyes sustantivas de cooperativas españolas. 


\subsection{Cooperativas de segundo grado que integren a cooperativas con distinto grado de protección}

Las CSG que asocien a cooperativas que sean protegidas y especialmente protegidas, además de los beneficios fiscales previstos en el art. 33 LRFC, ya enumerados, disfrutarán de la bonificación contemplada en el art. 34.2, «que se aplicará, exclusivamente, sobre la cuota íntegra correspondiente a los resultados procedentes de las operaciones realizadas con las cooperativas especialmente protegidas». Este precepto establece que, en el Impuesto sobre Sociedades, disfrutarán de una bonificación del $50 \%$ de la cuota íntegra.

Esto significa que las CSG serán tratadas, en términos generales, como cooperativas protegidas, aunque podrán aplicar la bonificación prevista para las especialmente protegidas a la parte proporcional de los resultados que provengan de sus operaciones con sus cooperativas socias que tengan este carácter.

\subsection{Otras bonificaciones aplicables a las cooperativas de segundo grado}

Junto a las ventajas fiscales específicas recogidas en la LRFC, en la medida en que la LIS les resulta aplicable como ya hemos comentado, pueden disfrutar también de los beneficios en ella regulados dentro del régimen general, así como de los contemplados dentro del régimen fiscal especial para las empresas de reducida dimensión. Esto parece entrar en contradicción con la finalidad para la que se crea una $\mathrm{CSG}$, que lo que pretende es, fundamentalmente, ganar dimensión para ser más competitiva.

En cualquier caso, la aplicación de las bonificaciones previstas en la LIS se podrá llevar a cabo siempre y cuando dichos beneficios no sean incompatibles con los establecidos en la LRFC.

\section{Los grupos cooperativos en el impuesto sobre sociedades}

Las CSG pueden actuar como sociedades cooperativas individuales o como grupo cooperativo. Actuarán como grupo en la medida que estén constituidas exclusivamente por sociedades cooperativas y las relaciones entre ellas se realicen por acuerdo paritario y libre.

La relación de dominio en un grupo de sociedades cooperativas tiene una dirección inversa respecto de los grupos consolidados de sociedades anónimas. Así, en estos últimos, el poder de decisión de la sociedad dominante cabecera del grupo sobre el resto de entidades se consigue en virtud de la participación que aquella sociedad tiene en el capital social de las dependientes, lo que permite su control y, por tanto, su poder de decisión sobre tales entidades dependientes. Por el contrario, en un grupo cooperativo es la cabecera del grupo quien está participada por el resto de entidades cooperativas del mismo, por lo que el control sobre éstas no se tiene en virtud de ninguna participación en el capital, sino por medio de disposiciones estatutarias que así lo prevean (Garrido Pulido, Puentes Poyatos y Navarro Heras, 2000: 77-78). 
La propia LCOOP establece que «las especiales características de las sociedades cooperativas han hecho necesaria la regulación del grupo cooperativo, con la finalidad de impulsar la integración empresarial de este tipo de sociedades, ante el reto de tener que operar en mercados cada vez más globalizados» (Exposición de Motivos).

Los grupos cooperativos al igual que los grupos de sociedades, pueden aplicar dos regímenes de tributación:

- $\quad$ El régimen de declaración individual, en el que cada sociedad del grupo registra en su contabilidad el gasto por IS que deberá calcularse en función de su excedente cooperativo y de las posibles diferencias permanentes que puedan presentar sus operaciones, reconociendo, en su caso, los impuestos anticipados o diferidos que puedan existir; y

- $\quad$ El régimen de declaración consolidada, según el cual el sujeto pasivo del impuesto es el propio grupo, ostentando la sociedad dominante, a efectos de gestión, liquidación y recaudación del impuesto su representación ante la Administración. No obstante, todas las sociedades que la integran:

a) Están igualmente sujetas a las obligaciones tributarias que se derivan del régimen de tributación individual, excepción hecha del pago de la deuda tributaria.

b) Responden solidariamente del pago de la deuda tributaria consolidada, excluidas las sanciones.

Este procedimiento de tributación fiscal consolidada regulada en los arts. 55 a 75 LIS, es de difícil aplicación al grupo cooperativo, pues las peculiares reglas de funcionamiento de las cooperativas impiden que los grupos cooperativos se puedan encuadrar perfectamente en este régimen de tributación (Peris García, 2002: 95).

En previsión de estas dificultades, la LRFC en su disposición final segunda, apartado tercero, facultó al Gobierno para dictar las normas necesarias para adaptar el régimen de tributación del beneficio consolidado de los grupos de sociedades a las cooperativas que, en virtud de sus reglas estatutarias, mantuvieran relaciones de vinculación en el ejercicio de sus actividades económicas. El mandato se concretó en el Real Decreto 1345/1992, de 6 de noviembre, por el que se dictan las normas aplicables a los grupos cooperativos que opten por la tributación consolidada, que sigue en vigor en virtud de la disposición final primera de la actual LIS.

Esta norma define a efectos fiscales el grupo consolidado como el conjunto formado por una entidad cabeza de grupo, que puede no ser cooperativa, y las cooperativas que tengan consideración de socias del mismo. Por tanto, se centra en las relaciones de vinculación en sus actividades empresariales y se aleja del criterio de participación en el capital, que resulta determinante respecto de otros grupos societarios.

No obstante, a efectos de nuestro estudio, nos interesa el caso de que la cabeza del grupo cooperativo sea una CSG. Los requisitos para que así sea son: uno, que en virtud de sus reglas estatutarias se le conceda el poder de decisión (art. 1.2 RD); y dos, que haya un compromiso de redistribuir solidariamente el excedente neto obtenido por cada una de las cooperativas integrantes (art. 1.3 y 4 RD). Estos requisitos pueden ser cumplidos por las CSG en virtud del contenido de los 
estatutos de la misma y de acuerdos parasociales, aunque lo normal en la constitución de CSG sea ceder menos poder a la entidad cabeza de grupo que se utilizará básicamente para la comercialización conjunta de los productos de las cooperativas socias.

La especialidad más sustancial del régimen de tributación consolidada de los grupos cooperativos se centra en el sistema de integración de cuotas íntegras, que sustituye al de integración de bases imponibles. Los grupos cooperativos no precisan determinar una base imponible consolidada de grupo, sino que cada una de las sociedades integrantes han de calcular la suya propia, centrándose la liquidación del impuesto en la fijación de la cuota íntegra consolidada positiva o, en su caso, del importe negativo. La razón de este tratamiento radica en la existencia de dos bases imponibles, una cooperativa y otra extracooperativa, y las diferencias del tipo tributario a aplicar, de ahí que la aplicación del sistema de consolidación de bases imponibles obligaría, con posterioridad, a optar por un tipo tributario concreto a aplicar a la base consolidada y, creemos que con acierto, se ha entendido preferible consolidar las respectivas cuotas tributarias, una vez aplicado por cada cooperativa el tipo tributario que respectivamente le corresponde (Ormaechea, 1993: 11).

Por tanto, según lo dispuesto en el RD 1345/1992, el particular sistema de consolidación requiere:

1. ${ }^{\circ}$ Que cada cooperativa integrante del grupo determina su base imponible de acuerdo con lo estipulado en la LRFC y la LIS (en lo no previsto por aquélla) realizando exclusivamente las eliminaciones por:

a) operaciones de traspaso de excedentes o beneficios entre las entidades del grupo que corresponden a retornos entre cooperativas,

b) ayudas económicas obligatorias asumidas y que deban prestarse entre sí que no se consideran ni partida deducible ni ingreso computable

c) resultados distribuidos por la entidad cabeza de grupo.

2. ${ }^{\circ}$ Que se determinen las cuotas, positivas o negativas, de cada una de las entidades aplicando a las bases imponibles positivas o negativas, los tipos de gravamen que correspondan, así como, en su caso, las correspondientes bonificaciones previstas en la LRFC.

3. ${ }^{\circ}$ Que se obtenga la cuota íntegra consolidada mediante la suma algebraica de los importes resultantes de las cuotas tributarias de cada una de las entidades del grupo. En el caso de que la suma anterior fuese negativa, podrá compensarse con las cuotas consolidadas de los de los períodos impositivos siguientes, con el límite del $70 \%$ de la cuota íntegra previa a su compensación. En todo caso, serán compensables en el período impositivo cuotas íntegras por el importe que resulte de multiplicar un millón de euros al tipo medio de gravamen de la entidad (en aplicación de lo previsto en el art. 24 LRFC con efectos para los períodos impositivos iniciados a partir de 1 de enero de 2015, en su redacción dada por la por la disposición final cuarta 3.1 LIS, modificada posteriormente por la disposición final sexta 7 de la Ley 34/2015, de 21 de septiembre, de modificación parcial de la Ley 58/2003, de 17 de diciembre, General Tributaria).

Las cuotas tributarias negativas de cualquier sociedad cooperativa que se hubieran producido en períodos impositivos anteriores a aquél en que dicha cooperativa tribute en régimen de declaración consolidada, serán compensables 
sólo con cuotas tributarias positivas de la propia sociedad cooperativa y hasta el límite de éstas.

Esta diferente aplicación del régimen de consolidación fiscal se ha basado tradicionalmente en la idea de que en los grupos cooperativos no existe consolidación de estados financieros y por ello no se ha exigido la consolidación fiscal (Peris García, 2002: 102). No obstante, en respuesta a la habilitación que incluía la disposición final cuarta de la LCOOP, ya en 2003 se elaboró una norma de adaptación del Plan de General Contabilidad a las cooperativas, con objeto de que éstas dispusieran de un marco contable capaz de responder a las exigencias que derivan de sus características propias. En la actualidad rige la Orden EHA/3360/2010, de 21 de diciembre, por la que se aprueban las normas sobre los aspectos contables de las sociedades cooperativas. En ella se establece en qué casos el grupo cooperativo vendrá obligado a formular las cuentas anuales y el informe de gestión consolidados. En consecuencia, el RD 1345/1992 sólo resultaría aplicable a los grupos cooperativos que no consoliden cuentas.

El régimen es opcional y, como tal, implica el acuerdo de las Asambleas generales de las sociedades cooperativas de base para su aplicación. Será la CSG la encargada de solicitar al Ministerio de Economía y Hacienda la aplicación de dicho Régimen, ostentado así la representación a efectos de gestión, liquidación y recaudación del impuesto ante la Administración Tributaria (art. 61.1.y 2 LIS).

La duración de aplicación de este régimen especial, será indefinida, en tanto se mantengan los requisitos (por aplicación del art. 61.5 LIS en ausencia de regulación específica). De acuerdo con el RD 1345/1992, son causas de pérdida del derecho de aplicación de este régimen fiscal:

El incumplimiento del compromiso de redistribuir el excedente neto obtenido

La realización por parte de la entidad cabeza de grupo, cuando no sea cooperativa, de actividades no comprendidas dentro de su objeto exclusivo.

Junto a estas causas expresamente recogidas en el reglamento, también resultan de aplicación las contenidas en el art. 73 LIS para el régimen general de consolidación fiscal (Peris García, 2002: 101).

Se hace preciso decir que el RD 1345/1992, que se aprobó en cumplimiento del mandato del legislador contenido en la LRFC, se ha quedado más que obsoleto. En efecto, entró en vigor cuando en Impuesto sobre Sociedades se regía por la Ley 61/1978, de 27 de diciembre, y ha sobrevivido a tres leyes posteriores: la Ley 43/1995, de 27 de diciembre, el Real Decreto Legislativo 4/2004, de 5 de marzo, por el que se aprobó el texto refundido de la Ley del Impuesto sobre Sociedades y la vigente Ley 27/2014, de 27 de noviembre y también a las modificaciones que ha sufrido la propia LRFC. Se hace necesario, pues, un nuevo texto que se adapte a la regulación actual del impuesto para las cooperativas.

\section{Conclusiones}

- En cuanto al régimen fiscal especial aplicable a las cooperativas:

Las cooperativas persiguen unos objetivos de política económica y social, fomento del empleo, desarrollo sostenible, apoyo a las actividades agrarias, acceso a los bienes de consumo necesario, búsqueda de igualdad de las mujeres, etc., que 
justifican sobradamente la adopción de medidas fiscales específicas. A ello hay que unir los límites en su actuación, sobre todo a la hora de decidir el destino de sus beneficios. No obstante, el régimen fiscal especial ya no es tan favorable, sobre todo porque la tributación de las sociedades de capital en España se ha aligerado hasta equipararse al de las cooperativas. En algunos casos, incluso, será más beneficioso para ellas aplicar el régimen general.

- $\quad$ En relación a la tributación de las cooperativas de segundo grado:

En términos generales, estas cooperativas reproducen el esquema de las de primer grado. Así, si incurren en alguna causa de las previstas en el art. 13 LRFC, pierden el derecho a la aplicación del régimen especial, pasando a tributar según el régimen general del Impuesto; si estuvieran constituidas por cooperativas protegidas o especialmente protegidas participan de los beneficios correspondientes a estas categorías; y si estuvieran asociadas simultáneamente cooperativas con distinto grado de protección, gozarán de los beneficios de cada tipo en proporción a las operaciones realizadas con cada una de las cooperativas asociadas.

- $\quad$ En lo que se refiere a los grupos cooperativos encabezados por una CSG:

A efectos fiscales, el grupo consolidado será el conjunto formado por una CSG cabeza de grupo y las cooperativas que tengan consideración de socias del mismo. El nivel de cesión de poder a la CSG que se requiere hace que esta opción sea poco frecuente, aunque posible. Determinada la existencia del grupo cooperativo a efectos fiscales, a la cabeza de grupo le corresponderá cumplir las obligaciones tributarias derivadas del régimen fiscal especial de declaración consolidada, sin perjuicio de que las cooperativas integrantes del grupo deban cumplir también con las obligaciones que les son propias. El régimen de tributación consolidada de los grupos cooperativos se caracteriza por el sistema de integración de cuotas íntegras, que sustituye al de integración de bases imponibles.

Respecto a la fiscalidad como instrumento para conseguir cooperativas más competitivas:

No podemos obviar que la posible existencia de ventajas fiscales puede motivar la cooperación y la concentración empresariales. Las empresas, a la hora de tomar decisiones en cuanto a su forma jurídica, tienen en cuenta la legislación tributaria junto a factores relativos al mercado (la globalización y sus retos) o a las dificultades propias de su estructura (debilidad financiera, problemas de dimensión, etc.).

Es por ello por lo que, si se apuesta por poner en marcha medidas que fomenten la integración y la potenciación de las cooperativas y de los grupos de base cooperativa y asociativa, como hace, por ejemplo, la LFIC, tales medidas deberían ir acompañadas por los incentivos fiscales que se requieran para hacer atractiva la intercooperación o, en su caso, la concentración de cooperativas.

\section{Referencias bibliográficas}

Alfonso Sánchez, R. (2000) La integración cooperativa y sus técnicas de realización: La cooperativa de segundo grado. Valencia: Tirant lo Blanch. 575 p.

Cano Ortega, C. (2015) Hacia la calificación como entidad asociativa prioritaria: las diferentes formas de integración de las cooperativas agroalimentarias. REVESCO. 
Revista de Estudios Cooperativos, No 118, pp. 60-85. DOI: 10.5209/rev_REVE.2015.n118.49064.

Embid Irujo, J.M. (2016) Introducción al Derecho de los grupos de sociedades. Comares: Granada. 288 p.

Embid Irujo, J.M. (1998) Problemas actuales de la integración cooperativa. Revista de Derecho Mercantil, No 227, Vol. I, pp. 7-36.

Gadea Soler, E. (2010) Crisis e intercooperación: las cooperativas de segundo o ulterior grado como instrumento de colaboración empresarial. Boletín de la Asociación Internacional de Derecho Cooperativo, $\mathrm{N}^{\circ} 44$, pp. 251-262.

Garrido Pulido, T., Puentes Poyatos, R. y Navarro Heras, E. (2007) Fiscalidad de las cooperativas de segundo grado. REVESCO. Revista de Estudios Cooperativos, $\mathrm{N}^{\circ}$ 91, pp. 77-10.

Ormaechea, J.M. (1993) Tributación consolidada de grupos cooperativos. Partida Doble, $\mathrm{N}^{\mathrm{o}} 43$, pp. 9-13.

Peris García, P. (2002) Los procesos de concentración y de integración cooperativa y su tributación: Especial referencia a las fusiones y a la creación de grupos cooperativos. Ciriec-España. Revista Jurídica de Economía Social y Cooperativa, № 13, pp. 69-109.

Rodrigo Ruiz, M.A. (2010) Consideraciones sobre el régimen fiscal de las cooperativas. Problemas actuales y líneas de reforma. Ciriec-España. Revista Jurídica de Economía Social y Cooperativa, No 69, pp. 9-25.

Rosembuj, T. (1988) Economía de la cooperativa de segundo grado. Deusto, Anuario de Estudios Cooperativos, № 1, pp. 166-182.

Tejerizo López, J.M. (2008) El Régimen Tributario de las Cooperativas en España. Aspectos Generales. Gezki, No 4, pp. 41-78.

Vargas Vasserot, C, Gadea Soler, E y Sacristán Bergia, F. (2017) Derecho de las Sociedades Cooperativas. Madrid: La Ley. 434 p. 